\title{
LETTERS
}

\section{The utility and value of the "surprise question" for patients with serious illness}

Clinicians have long identified the challenges of accurately making a prognosis as being a barrier to patient access to end-of-life care, ${ }^{1}$ and many tools have been developed to help clinicians with the process. ${ }^{2}$ These tools generally rely on algorithms that can be complex and not easily used in a clinical setting, but as noted in a recent systematic review by Downar and colleagues, ${ }^{3}$ the surprise question ("Would I be surprised if this patient were to die within the next 12 months?") has gained support as a simple, practical way of identifying patients at high risk of dying.

However, the researchers found that the studies examining the prognostic accuracy of the surprise question have had mixed results, leading to the conclusion that it is not well suited as an indication of prognosis. This may be true, but does that mean the surprise question lacks clinical or research value?

Before throwing the surprise question out with the proverbial bath water, one must consider the origin of the surprise question. It was never intended to be a prognostic indicator, much less a standalone one. As originally conceived, the surprise question aimed to identify those who needed attention with respect to issues of decline and death, not just those who were surely going to die within a specified time frame. The question deliberately inverted the often unstated implication of the hospice question, which could be phrased, "Does this patient's situation make it overwhelmingly likely that this person will die within six months?" Or, more colloquially, "Does this patient promise to die on time?" The "surprise question" taps into the clinician's awareness that the patient's situation is not likely to end well, no matter how long it takes. The patient has serious illnesses and little resilience; and, although some in this situation will hang on for some years, throughout whatever time the person gets, he or she will be in fragile health.
Downar and colleagues suggest that because the surprise question has a high false-positive rate as a prognostic indicator, it may not be a suitable trigger for referrals to hospice or palliative care. ${ }^{3}$ The former may be true, but the same cannot be said for the latter. Putting hospice and palliative care together as being only appropriate for the end-of-life care is misleading. If clinicians believe their patients are at serious risk of dying within 12 months, they are relying on a clinical understanding of patients' capabilities and needs; thus, additional support to patient and family and additional planning are appropriate and the need for monitoring is prudent, even without a hospice referral. In addition, palliative care is widely considered to be appropriate across the continuum of advanced, serious illness; ${ }^{4}$ consequently, providing palliative care would generally precede eligibility for hospice.

A substantial limitation of all the studies analyzed in the meta-analysis done by Downar and colleagues is that the studies did not describe the health status among those who did not die in the prescribed time frame and for whom the answer to the surprise question was that dying within a year would not be surprising. The question remains: what were the longer-surviving participants' needs? Quite likely, virtually all of these participants continued to live with the consequences of serious advanced illness and had complex needs that supportive services could address. They also probably stayed very close to dying throughout, but luck averted the crises that might have caused death.

The surprise question has been used in conjunction with clinical criteria to determine whether patients are appropriate for additional services, as with the Gold Standards Framework in England (www.goldstandardsframework.org.uk/ PIG). The Gold Standards Framework's Proactive Indicator Guidance has effectively identified these patients, but clinical guidance may also improve the prognostic accuracy of the surprise question, especially when the clinician is unsure of the response. ${ }^{5,6}$ So, the question may need to be, "Would I be surprised if this patient were to die within a year, and if the response is 'uncertain,' has he or she experienced a clinical change or decline recently?"

Still, the focus on prognostic accuracy may be doing a disservice to clinicians, researchers and patients. Although many clinicians may wish they had a reliable, accurate way to predict short-term prognosis, it is probably unrealistic to expect to find one that can give a highly specific and sensitive answer. The final stage of life is unpredictable and uncertain, particularly in this age of high-tech medicine, which has enabled clinicians to intervene and extend life when death was once a certainty. Indeed, the reduced exposure to infectious agents and better diet and supportive care may well be major factors in allowing many people with serious and advanced illnesses to stay alive for long periods, despite functional limitations and lack of reserve in many body functions.

The transition to the end of life for people slowly declining with dementia, motor neuron diseases, organ system failures and other largely degenerative illnesses will continue to be indistinct. The "surprise" question helps clinicians to recognize when patients may have entered this transition so that appropriate services can be provided. Researchers can use the surprise question to identify these patients, explore their experiences and needs across the full length of their living "in the shadow of death," and thereby inform clinical care. The patient whose situation would make it no surprise if he or she died within a year should have an in-depth assessment and highly reliable and comprehensive services available for however long he or she lives, be it a few weeks or a few years. Consequently, the surprise question remains relevant.

\section{Rafael D. Romo PhD PHN}

Assistant Professor, University of Virginia School of Nursing, Charlottesville, Va.

\section{Joanne Lynn MD}

Director, Center for Elder Care and

Advanced Illness, Altarum Institute, Washington, DC 
Cite as: CMAJ 2017 August 21;189:E1072-3. doi: $10.1503 /$ cmaj. 733231

\section{References}

1. Brickner L, Scannell K, Marquet S, et al. Barriers to hospice care and referrals: survey of physicians' knowledge, attitudes, and perceptions in a health maintenance organization. J Palliat Med 2004;7:411-8.
2. Yourman LC, Lee SJ, Schonberg MA, et al. Prognostic indices for older adults: a systematic review. JAMA 2012;307:182-92.

3. Downar J, Goldman R, Pinto R, et al. The "surprise question" for predicting death in seriously ill patients: a systematic review and meta-analysis. CMAJ 2017;189:E484-93.

4. National Consensus Project for Quality Palliative Care. Clinical practice guidelines for quality palliative care. 2nd ed. Pittsburgh: National Consensus Project for Quality Palliative Care; 2009.
5. Fenning S, Woolcock R, Haga K, et al. Identifying acute coronary syndrome patients approaching end-of-life. PLoS One 2012;7:e35536.

6. O'Callaghan A, Laking G, Frey R, et al. Can we predict which hospitalised patients are in their last year of life? A prospective cross-sectional study of the Gold Standards Framework Prognostic Indicator Guidance as a screening tool in the acute hospital setting. Palliat Med 2014;28:1046-52. 